\title{
NEUROPSYCHOLOGICAL PREDICTORS OF SAFETY IN URBAN LEFT-TURN SCENARIOS
}

\author{
Jeffrey D. Dawson ${ }^{1}$, Lixi Yu ${ }^{1}$, Kuan-Hua Chen $^{2}$, Michelle Rusch ${ }^{2}$, \\ Amy M. Johnson ${ }^{1}$, Nazan S. Aksan ${ }^{2}$, Takashi Sunda ${ }^{3}$, Machiko Hiramatsu ${ }^{3}$, \\ Steven W. Anderson ${ }^{2} \&$ Matthew Rizzo ${ }^{2}$ \\ ${ }^{1}$ Dept. of Biostatistics, Univ. of Iowa College of Public Health, Iowa City, Iowa, USA \\ ${ }^{2}$ Dept. of Neurology, Univ. of Iowa Carver College of Medicine, Iowa City, Iowa, USA \\ ${ }^{3}$ Nissan Research Center, Nissan Motor Co., Ltd., Japan \\ Email: jeffrey-dawson@uiowa.edu
}

Summary: Left turns at urban intersections can be dangerous, especially when views are obstructed or pedestrians are present. Impairments in driver vision, motor, and cognition functions may further increase left-turn risk. We examined this problem in a simulated environment that included left-turn scenarios to study the driving behaviors of 28 drivers, ages 37 to 88 years, six of whom had "Useful Field of View" (UFOV) impairments. Subjects also completed a battery of neuropsychological tests. The simulated drive included an urban section with six left turns in three types of scenarios: 1) a semi truck blocking the view of oncoming traffic, 2) a lead vehicle obstruction, and 3) a pedestrian crossing ahead of the turning driver. Results showed a mean (SD) of 1.46 (1.60) collisions per driver (range 0 to 7 ), $83 \%$ of which occurred at intersections with semi trucks. Far visual acuity, contrast sensitivity, UFOV, Mini Mental State Examination, TrailMaking Test Part B, the Wisconsin Card Sort task, and age were all associated with the total number of collisions (Pearson correlation magnitudes between 0.37 to 0.77 ; p-values $<0.05)$. Spearman correlations were less significant. Findings indicate that visual obstruction by on oncoming semi-truck is a particularly dangerous left-turn situation.

\section{INTRODUCTION}

Making a left turn at urban intersections without turn signals (unprotected intersections) can be dangerous, especially when there are obstructed views or pedestrians present. Nearly one fifth of all vehicle crashes in the United States occur during left turns across opposing streams of traffic (Chan et al., 2005). Impairments in vision, motor skills, and cognition are ubiquitous in the general population, increase with age, and may further increase the safety risk (Dawson et al, 2009). Older drivers are especially at risk (Chandraratna \& Stamatiadis, 2003; Gelau, 2009; Mayhew, Simpson, \& Ferguson, 2006) most likely due to age-related perceptual, cognitive, and motor dysfunction (Kausler, 1991). Specifically, impairments associated with divided attention (Dewar, 2002; Hakamies-Blomqvist et al, 1996), visual search (Ho, Scialfa, Caird, \& Graw, 2001; Maltz \& Shinar, 1999), and UFOV (Ball \& Owsley, 1991), an index of speed of processing for visual attention, may negatively affect a driver's ability to detect oncoming vehicles. Consequently, we developed several challenging simulated driving scenarios to study the driving safety of middle-aged and elderly drivers making left hand turns under challenging circumstances. The purpose of our study was to assess how often drivers experienced collisions, 
to compare specific left-turn scenario types, and to test the hypothesis that collisions are associated with neuropsychological factors.

\section{METHODS}

\section{Subjects}

Subjects included 16 middle aged drivers (37 to 56 years old) and 12 elderly ( $>70$ years old). Elderly drivers were evenly split between being impaired and unimpaired in terms UFOV total score. Participants included 14 men and 14 women.

\section{Visual and Neuropsychological Test Battery}

These standardized tests were selected based on relevance to driving and brain function. Vision tests included near and far visual acuity (in $\log$ MAR scale, which is log base 10 of the Minimal Angle of Resolution) and contrast sensitivity. The neuropsychological tests included the UFOV (total loss summed across four subtests), Mini Mental State Examination (MMSE), Trail Making Test Part B (time in seconds), and the Wisconsin Card Sorting Task (WCST, preservative errors and categories completed). For test descriptions, see Ball et al (1993), Lezak, Howieson \& Loring (2004), and Strauss, Sherman \& Spreen (2006). Note that high scores indicate better function for contrast sensitivity, MMSE, and WCST categories completed, while high scores show impairment for the other tests. All of these test scores were considered as predictors, as were two demographic variables: age and driving frequency.

\section{Collecting Driving Data}

This study used a NADS MiniSim ${ }^{\mathrm{TM}}$, desktop-based driving simulator developed by National Advanced Driving Simulator (NADS) and the University of Iowa. The simulator had three 20" display channels, spanning a $110^{\circ}$ front-view. We designed a drive comprising an urban section followed by a rural section.

The urban section contained 13 intersections, 12 of which required the driver to turn. Six intersections required a left turn, which were evenly divided among three scenario types. "Type 1: Semi" scenarios involved an oncoming semi truck that was stopped in the intersection, waiting to make a left turn. It was difficult for the driver to see other oncoming vehicles that were bypassing the truck (see Figure 1, upper panel). In "Type 2: Lead Vehicle" scenarios (Figure 1, middle panel), a vehicle in front of the subject was also turning left, obstructing the view of oncoming vehicles. If the subject followed the lead vehicle too closely through the intersection, instead of waiting for an unobstructed view to evaluate oncoming traffic, there was a high risk of a collision. In "Type 3: Pedestrian" scenarios, a runner crossed directly in front of the vehicle of the driver completing the left turn (Figure 1, lower panel). If the driver was not vigilant in checking the left screen of the simulator, there was a risk of colliding with the pedestrian. In all of these left turns, there were no green arrows to assign right of way for a turning vehicle. 


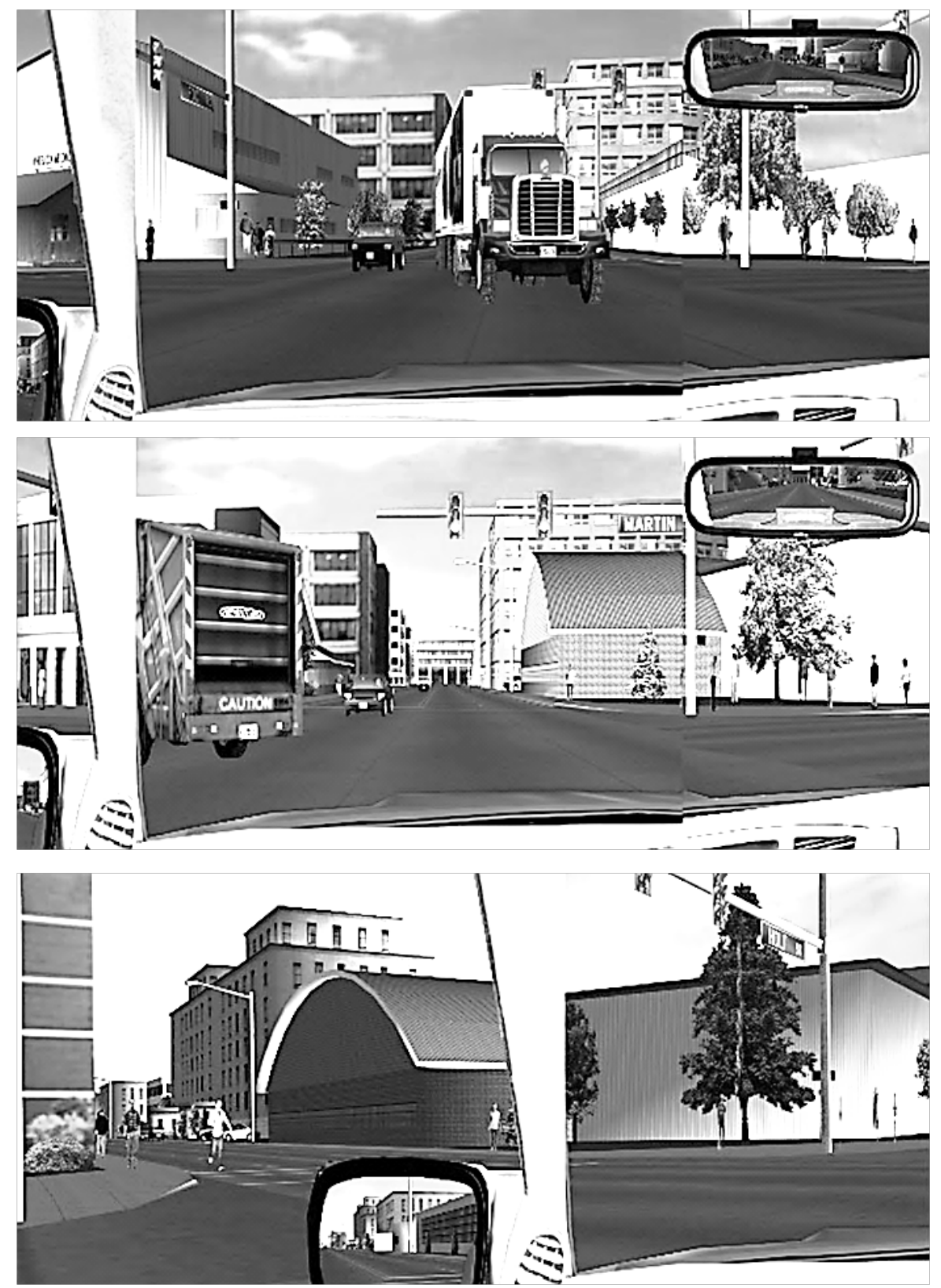

Figure 1. Views of the Three Scenario Types. From Top to Bottom, Type 1: Semi, Type 2: Lead Vehicle, and Type 3: Pedestrian

Ambient traffic, background pedestrians, and police patrol cars were included in all scenarios to increase scenario complexity and reality. Subjects were instructed to drive as they would in the real world and to follow the rules of the road, e.g., obeying speed limits, yielding to pedestrians and other vehicles, etc. The entire drive generally took 25-30 minutes (15-20 minutes for urban driving), not including two or three brief practice segments before the official drive to help subjects adapt to the simulator. The subjects were allowed a 3-5 minute break between the urban and rural section, if requested. 


\section{Statistical Analysis}

Descriptive statistics (mean, SD, minimum, median, and maximum) of neuropsychological variables were calculated, and the number of collisions was calculated for each scenario type, as well as for the entire drive. The Wilcoxon Signed-Rank test was used for comparing the number of crashes across the three scenario types. Pearson and Spearman correlations were calculated for predicting total collisions using demographics, visual, and neuropsychological variables. For additional insight, scatter plots were created, with linear regression fits and confidence bands superimposed.

\section{RESULTS}

Table 1 displays the descriptive statistics of the demographics and neuropsychological predictors (cognitive and vision tests). For the entire drive, the mean (SD) of collisions per driver was 1.46 (1.60), with range 0 to 7. Eighty-three percent of the collisions occurred during the Type 1: Semi intersections, where the mean (SD) was 1.21 (1.17), with range 0 to 7 . The rate of collisions in Type 1: Semi intersections was higher than in the other two left-turn scenarios $(\mathrm{p}<0.0001$ in both cases), where no collisions occurred.

Table 1. Descriptive Statistics of Demographics, Vision, and Neuropsychological Predictors

\begin{tabular}{lccc}
\multicolumn{1}{c}{ Variable } & $\mathrm{N}$ & Mean \pm standard deviation & Minimum, Median, Maximum \\
\hline Demographics & 28 & $59.75 \pm 16.92$ & $37.00,53.00,88.00$ \\
Age (years) & 28 & $5.86 \pm 1.46$ & $2.00,6.50,7.00$ \\
Driving frequency (days/wk) & & & $0.00,0.01,0.22$ \\
\hline Vision tests & 28 & $0.05 \pm 0.07$ & $-0.18,0.00,0.32$ \\
Near Visual Acuity (logMAR scale) & $0.01 \pm 0.16$ & $0.90,1.70,1.95$ \\
Far Visual Acuity (logMAR scale) & 28 & $1.64 \pm 0.22$ & $145.00,352.00,1516.00$ \\
Contrast sensitivity & 28 & & $27.00,30.00,30.00$ \\
\hline Neuropsychological tests & & $444.46 \pm 299.31$ & $36.87,64.66,161.25$ \\
UFOV total loss & 28 & $29.54 \pm 0.74$ & $4.00,6.00,49.00$ \\
MMSE & 28 & $72.02 \pm 31.38$ & $1.00,6.00,6.00$ \\
Trail-Making Test Part B (sec) & 28 & $9.26 \pm 8.99$ & $5.67 \pm 1.07$ \\
WCST (perseverative errors) & 27 & & \\
WCST (categories completed) & 27 & & \\
\hline
\end{tabular}

Results showed that age, far visual acuity, contrast sensitivity, UFOV, MMSE, Trail-Making Test Part B, and the Wisconsin Card Sort task were all associated with the total number of collisions (Pearson correlation magnitudes between 0.37 to 0.77 ; p-values ranging from 0.0489 to $<0.0001$ ). Spearman correlations tended to be lower and less significant. In particular, MMSE was no longer even close to significant $(\mathrm{p}=0.2592)$, while age and contrast sensitivity decreased to near-significance ( $\mathrm{p}=0.0848$ and 0.0679 , respectively). See Table 2 for details. Note that all significant and near-significant correlations were in the anticipated direction (i.e., worse scores being associated with more crashes). 
Table 2. Correlations (and P-Values) Between Predictors and Total Collisions

\begin{tabular}{lcc}
\hline \multicolumn{1}{c}{ Predictor Variable } & Pearson & Spearman \\
\hline Demographics & $0.37(0.0489)$ & $0.33(0.0848)$ \\
Age (years) & $0.06(0.7567)$ & $0.16(0.4211)$ \\
Driving frequency (days/wk) & $-0.06(0.7456)$ & $-0.09(0.6555)$ \\
\hline Vision tests & $0.40(0.0331)$ & $0.38(0.0448)$ \\
Near Visual Acuity (logMAR scale) & $-0.47(0.0118)$ & $-0.35(0.0679)$ \\
Far Visual Acuity (logMAR scale) & & \\
Contrast sensitivity & $0.47(0.0121)$ & $0.38(0.0457)$ \\
\hline Neuropsychological tests & $-0.53(0.0039)$ & $-0.22(0.2592)$ \\
UFOV total loss & $0.64(0.0002)$ & $0.40(0.0333)$ \\
MMSE & $0.77(<0.0001)$ & $0.42(0.0277)$ \\
Trail-Making Test Part B (sec) & $-0.72(<0.0001)$ & $-0.39(0.0460)$ \\
WCST (perseverative errors) &
\end{tabular}

The reason for the discrepancies can be seen in Figure 2, which shows how MMSE and WCST perseverative errors relate to crashes. One subject had extreme values of collisions (with 7), MMSE (27), and WCST errors (49). The Spearman approach down-weights that data, rendering MMSE a non-significant predictor of crashes and WCST errors less statistically significant.
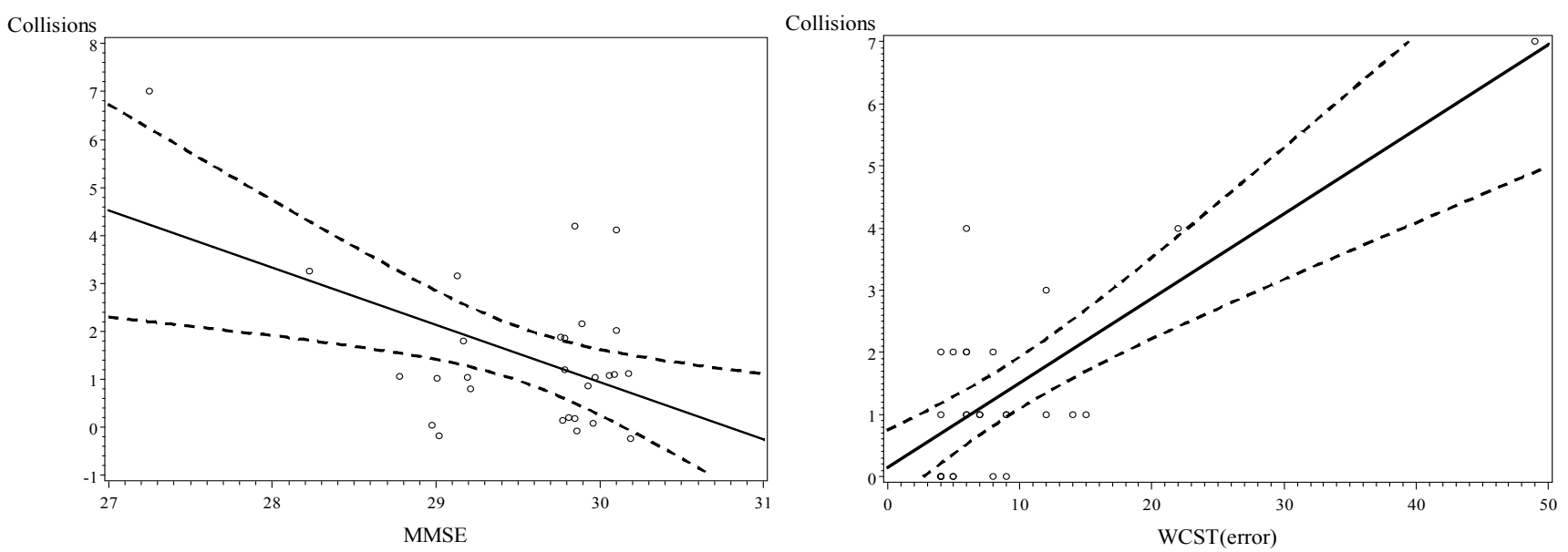

Figure 2. Scatter plots, linear fit, and 95\% confident bands of MMSE and WCST predicting total collisions

\section{DISCUSSION}

In this study of middle aged and elder drivers, we found that having an oncoming semi truck obstructing the view of oncoming traffic was particularly hazardous. By contrast, the lead vehicle scenario did not trigger driver tailgating behavior to increase the risk of crashes into oncoming traffic. Similarly, the drivers could avoid the running pedestrian in all cases. Several 
neuropsychological scores were associated with total number of crashes, but some of the significance was due to one driver with impairments in multiple domains who had seven crashes.

Older drivers may be at higher risk during left turns because they make poor judgments in selecting appropriate time gaps (Andersen \& Enriquez, 2006). They typically choose only large gaps or wait for traffic to clear entirely to compensate for difficulties they have in estimating the speed and distance of oncoming traffic (e.g. Caird \& Hancock, 2002; Skaar, Rizzo, \& Stierman, 2003). In our scenarios, where views were obstructed and the traffic was non-stop, this strategy was not an accessible option. Many drivers waited for an extended period of time and then eventually turned into an unsafe gap between oncoming vehicles. This resulted in imperfect and erratic turns. The ability to avoid collisions with the pedestrian may reflect greater emotional valence and greater signal value of a moving human target. Our simulated scenarios were developed and calibrated to have a high probability of collisions. Hence, our results do not imply that drivers are likely to crash several times in a 30-minute drive in the real world. Also, we acknowledge that drivers can behave differently in a simulator where no injury can occur.

In future analyses, we plan to investigate the kinematics of the left hand turns, based on the electronic data from the simulator. From these future analyses, we hope to gain an understanding of left turns that resulted in collisions, as well as those that did not. The emerging findings may inform the development and settings of in-vehicle safety systems, including alerting and warning signals, and communication systems (e.g., vehicle to vehicle and driver to driver) for improving the situation awareness, turn-taking, and conflict resolution among drivers at intersections.

\section{ACKNOWLEDGMENT}

This study was supported by Nissan Motor Company. We thank Katherine Read, Kelsey Thompson, Samantha Edwards, Mark Schall, and Ruth Henson for their collaborative assistance. We also thank Andrew Veit, Chris Schwarz, David Heitbrink, Omar Ahmad, and Meiji Zhang in the National Advanced Driving Simulator for their help with the MiniSim ${ }^{\mathrm{TM}}$ hardware and software. We also thank all of our subjects for their participation.

\section{REFERENCES}

Andersen, G. J., \& Enriquez, Z. (2006). Aging and the detection of observer and moving object collisions. Psychology of Aging, 21, 74-85.

Ball, K., \& Owsley, C. (1991). Identifying correlates of accident involvement for the older driver. Human Factors, 33, 583-595.

Ball, K., Owsley, C., Sloane, M.E., Roenker, D.L., \& Bruniw, J.L. (1993). Visual attention problems as a predictor of vehicle crashes in older drivers. Investigative Ophthalmology \& Visual Science, 34, 3110-3123.

Caird, J. K., \& Hancock, P. A. (2002). Left-turn and gap acceptance crashes. In Dewar, R. E., \& Olson, P. (Eds). Human factors in traffic safety. Tucson, AZ: Lawyers \& Judges Publishing. 
Chan, C., Ragland, D. R., Shladover, S. E., Misener, J. A., \& Marco, D. (2005). Observations of driver time gap acceptance at intersections in left-turn across-path-opposite-direction scenarios. Transportation Research Record, 1910, 10-19.

Chandraratna, S., \& Stamatiadis, N. (2003). Problem driving maneuvers of elderly drivers. Transportation Research Record, 1843, 89-95.

Dawson, J. D., Uc, E. Y., Anderson, S. W., Johnson, A. M., \& Rizzo, M. (2010). Neuropsychological predictors of driving errors in older adults. Journal of the American Geriatrics Society, 58, 1090-1096.

Dewar, D. E. (2002). Age difference: drivers old and young. In D. E. Dewar \& P. Olson (Eds.), Human factors in driving safety. Tucson, AZ: Lawyers \& Judges Publishing.

Gelau, C. (2009). Elderly drivers' needs for support at intersections. In M. W. Greenlee (Ed.), New issues in experimental and applied psychology: A festschrift for alf zimmer. Lengerich, Germany: Pabst-Science-Publishers.

Hakamies-Blomqvist, L., Johansson, K., \& Lundberg, C. (1996). Medical screening of older drivers as a traffic safety measure: a comparative Finnish-Swedish evaluation study. Journal of the American Geriatrics Society, 44, 650-653.

Ho, G., Scialfa, C. T., Caird, J. K., \& Graw, T. (2001). Visual search for traffic signs: the effects of clutter, luminance, and aging. Human Factors, 43, 194-207.

Kausler, D. (1991). Experimental psychology, cognition and human aging. New York: Springer.

Lezak, M.D., Howieson, D.B., \& Loring, D.W. (2004). Neuropsychological Assessment (4th ed.). New York: Oxford University Press.

Maltz, M., \& Shinar, D. (1999). Eye movements of younger and older drivers. Human Factors, $41,15-25$.

Mayhew, D. R., Simpson, H., \& Ferguson, S. A. (2006). Collisions involving senior drivers: High-risk conditions and locations. Traffic Injury Prevention, 7, 117-124.

Skaar, N., Rizzo, M., \& Stierman, L. (2003). Traffic entry judgments by aging drivers. Presented at: Second International Driving Symposium on Human Factors in Driver Assessment, Training and Vehicle Design, July23, 2003; Park City, Utah.

Strauss, E., Sherman, E.M.S., \& Spreen, O. (2006). A Compendium of Neuropsychological Tests: Administration, Norms, and Commentary ( $4^{\text {th }}$ ed.). New York: Oxford University Press. 